\title{
Frequency of acute antibody mediated rejection in renal allograft biopsies as detected by morphological findings and C4d immunostaining
}

\author{
Hina Tariq ${ }^{*}$, Humaira Nasir \\ Histopathology Department, Shifa International Hospital, Islamabad, Pakistan
}

\section{A R T I C L E I N F O}

Article Type:

Original

\section{Article History:}

Received: 3 June 2017

Accepted: 14 December 2017

Published online: 3 January 2018

\section{Keywords:}

Acute antibody-mediated rejection, Complement C4d, Chronic antibody-mediated rejection, Renal allograft biopsies, Morphological findings, Immunofluorescence, End-stage renal disease

\begin{abstract}
A B S T RACT
Introduction: Acute antibody-mediated rejection (AMR) detection using C4d immunofluorescence in combination with histopathological examination in renal allograft biopsy is a gold standard for AMR. This study will be the first one in this context in Pakistan and will generate local data.

Objectives: To determine the frequency of AMR in renal allograft biopsies as detected by morphological findings and $\mathrm{C} 4 \mathrm{~d}$ immunostaining.

Patients and Methods: We performed a prospective cross-sectional study at histopathology department, Shifa international hospital, Islamabad. Allograft biopsies which were performed for diagnosis of graft dysfunction were included. Interpretation was performed, according to Banff 2013. All statistical analysis of the data was performed using statistical software SPSS 20.

Results: A total of 60 biopsies, including 59 (98.3\%) males and 1 (1.7\%) female were evaluated. Age range was 15-65 years (mean $34.6 \pm 11.8$ ). Out of 60 biopsies, in the early post-transplant period, 14 (23.3\%) out of 60 cases were diagnosed as AMR on morphology. One (1.6\%) case had strong C4d positive in absence of morphological features. Out of these cases, 8 (57.1\%) showed C4d positivity, 2 (14.3\%) showed focal C4d positivity while 4 (28.6\%) were C4d negative. In the late post-transplant period, chronic allograft nephropathy (CAN) cases were $19(31.7 \%)$. Out of these cases, 13 (68.4\%) showed morphological features of AMR. C4d was positive 11 (57.9\%) and C4d was negative in 2 cases (10.5\%).

Conclusion: C4d positivity favors AMR; however, it can be seen in absence of morphological features.
\end{abstract}

Implication for health policy/practice/research/medical education:

Acute antibody-mediated rejection (AMR) has emerged as an important reason of allograft dysfunction. Histopathologic assessment of post-transplant renal biopsies was routinely performed in many canters for AMR. C4d has emerged as an easy and inexpensive means of detection of AMR. With its help in combination with histopathological findings, AMR can be timely diagnosed. These patients could be benefited from anti-humoral therapy and prevented from more intense rejection in future.

Please cite this paper as: Tariq H, Nasir H. Frequency of acute antibody mediated rejection in renal allograft biopsies as detected by morphological findings and C4d immunostaining. J Renal Inj Prev. 2018;7(3):189-196. doi: 10.15171/jrip.2018.45.

\section{Introduction}

In South Asia, true prevalence of end-stage renal disease (ESRD) patients in Pakistan and India are not established due to lack of national registries. Chronic glomerulonephritis and diabetic nephropathy are leading causes of ESRD patients in India. Patients are younger at the time of detection of $\operatorname{ESRD}(1,2)$.
Around 5\% of all ESRD patients undergo transplant. In India, live related transplants constitute $30 \%-40 \%$ and cadaveric transplantation account for less than $2 \%$ (2). Life expectancy of these patients has prolonged due to availability of organ transplantation (3-5). Acute antibodymediated rejection (AMR) has emerged as an important reason of allograft dysfunction. It usually occurs a few 
weeks post-transplant and is mediated by antibodies to donor epithelium (6). C4d detection was not possible for many years, as polyvalent anti-C4 reagent available at that time, reacted only with determinants of the $\mathrm{C} 4 \mathrm{c}$ fragment, but not with the alpha-2 portion of C4 (7). Over last 2 decades, the incidence of acute rejection has reduced due to novel immunosuppressive drugs. The incidence of rejection episodes in whole series reported by Pallardó Mateu et al was $32.5 \%$ (8).

The incidence of acute AMR in un-sensitized patients is $<5 \%$, but it can reach $40 \%-90 \%$ among sensitized patients. AMR has a major impact on long-term graft survival (9). Allograft dysfunction in C4d-positive rejection episodes is often more pronounced. These patients benefit from intensive therapy, potentially preventing the previously reported high graft failure rate (10). Histopathologic assessment of post-transplant renal biopsies was routinely performed in many centers. Detection of C4d in renal allograft biopsy by IF in combination with routine histopathological examination is now the gold standard for $\operatorname{AMR}(6,11,12)$.

Banff group of nephrologists and transplant pathologists is leading body for updating classification of renal allograft rejection. C4d was incorporated in Banff classification in 2005 (13). In Banff 2007 peritubular capillaritis grading, C4d scoring, interpretation of C4d deposition without morphological evidence of active rejection and scoring of total interstitial inflammation (ti-score) were defined $(14,15)$. Banff criteria for AMR were refined in June 2011 with inclusion of C4d negative AMR. At that time multicenter data was analyzed, however it was decided to collect more data and further research about it (16). Various studies from literature can be documented from 2007 onwards to support the role of C4d immunostaining for early detection of AMR. In this regard, a study conducted on 59 cases of acute renal rejection by Ranjan et al shows $55 \%$ of cases of acute rejection with C4d positivity. Out of these, $81 \%$ were of presumptive antibody mediated rejection P-AbAR/ AMR (6).

Analysis of C4d immunostaining shows agreement quite well with various histopathological findings for AMR (11). Comparing renal transplant recipients with and without AMR, patients exhibiting subclinical AMR (C4d positivity and capillaritis/glomerulitis) in their first biopsy were found to be at an increased risk of more intense chronic lesions in their follow-up biopsies (17).

C4d has emerged as an easy and inexpensive means of detection of AMR. With its help in combination with histopathological findings, AMR can be timely diagnosed. These patients could be benefited from anti-humoral therapy and prevented from more intense rejection in the future $(2,18)$.

Despite the emergence of various molecular techniques, like panel reactive antibody (PRA) levels, flow cytometry cross-match and flow PRA bead assays, biopsy remains gold standard for detection of AMR $(6,11-13,19)$.
Renal transplant is being performed in Shifa international hospital, since last 15 years. Previously only histopathological assessments of biopsies were done to look for rejection. Now $\mathrm{C} 4 \mathrm{~d}$ is introduced in routine practice and will be a great help in the early diagnosis and treatment of patients affected with AMR. This study will be the first study in this context in Pakistan and will generate local data.

\section{Objectives}

To determine the frequency of AMR in renal allograft biopsies as detected by morphological findings and $\mathrm{C} 4 \mathrm{~d}$ immunostaining.

\section{Patients and Methods}

\section{Study and setting}

We performed a prospective cross-sectional study at histopathology department, Shifa international hospital, Islamabad.

\section{Definition of acute antibody mediated rejection Clinical and laboratory data}

Allograft biopsies which were performed for diagnosis of graft dysfunction were included. Non-probability, consecutive sampling was done. For histopathological assessment biopsy with more than 7 glomeruli and 1 or more arteries was included. Biopsy with no glomeruli or no arteries or with only medulla was considered nondiagnostic. For immunofluorescence, renal biopsy with or without glomeruli was included.

\section{Preparation of biopsy sample}

Using WHO sample size calculator, keeping confidence level 95\%, anticipated population proportion $81 \%$ (6), and absolute precision required $10 \%$, sample size is 60 . Patient's medical record number age and gender patient were retrieved from patient record file or by telephone communication. Patient's confidentiality was maintained at each step of the research. Two transplant renal biopsy specimens were received from each patient, one in formalin for routine microscopy and other in transplant media (Zeus Scientific Inc., Branchburg, NJ, USA), for immunofluorescence.

\section{Ethical issues}

The research followed the tenets of the Declaration of Helsinki; informed consent was obtained; and the research was approved by the ethical committee of Shifa international hospital, Islamabad, Pakistan. All patients' information remained confidential.

\section{Statistical analysis}

Interpretation was performed according to Banff 2013 for assessment of AMR. All statistical analysis of the data was performed using SPSS version 20. Percentages, means and standard deviations were calculated. 


\section{Results}

A total of 60 biopsies, including 59 (98.3\%) males and $1(1.7 \%)$ female were evaluated (Figure 1). Age range was $15-65$ years (mean $34.6 \pm 11.8$ ) (Table 1 ). Out of 60 biopsies, in the early post-transplant period $14(23.3 \%)$ out of 60 cases were diagnosed as AMR on morphology. Out of these cases $8(57.1 \%)$ showed C4d positivity, 2 (14.3\%) showed focal C4d positivity while 4 (28.6\%) were C4d negative. One case had strong C4d positivity in absence of morphological features (Figure 2). In the late post-transplant period, chronic allograft nephropathy (CAN) cases were 19 (31.7\%). Out of these cases, 13 (68.4\%) showed morphological features of AMR. C4d was positive $11(57.9 \%)$ and C4d was negative in $2(10.5 \%)$ (Figure 3). Morphological and C4d patterns in AMR with and without CAN are illustrated in Figure 4A-H.

Rest of biopsies show additional pathological features. ACR was documented in $7(11.6 \%)$ cases, CNI toxicity in $5(8.3 \%)$ cases, TIN in $5(8.3 \%)$ cases, tubular damage and necrosis $5(8.3 \%)$ cases. In 2 of the above-mentioned cases of AMR, morphological features of both ACR and AMR were present. BK nephropathy was documented

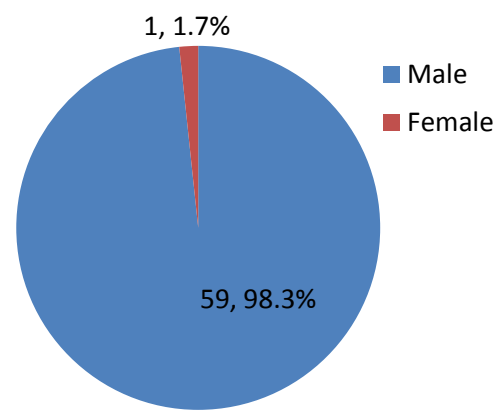

Figure 1. Gender distribution.

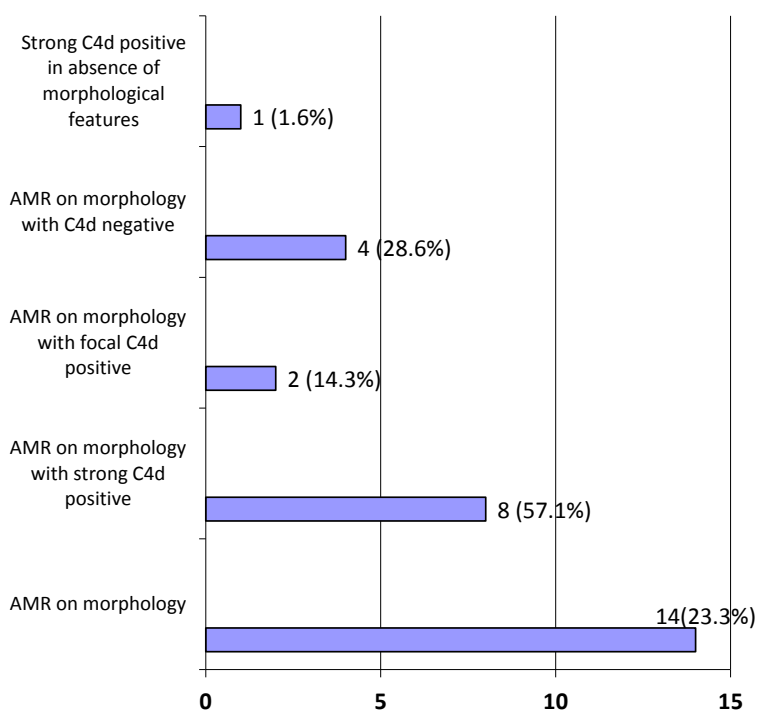

Figure 2. Distribution of acute antibody mediated rejection in early period of post transplantation.
Table 1. Range, mean and standard deviation of age and duration of post-transplant

\begin{tabular}{ll}
\hline Variables & Results \\
\hline Age & $15-65$ y $(34.6 \pm 11.8)$ \\
Duration Post-transplant & 3 days - 180 months $(33.8 \pm 46.8)$ \\
\hline
\end{tabular}

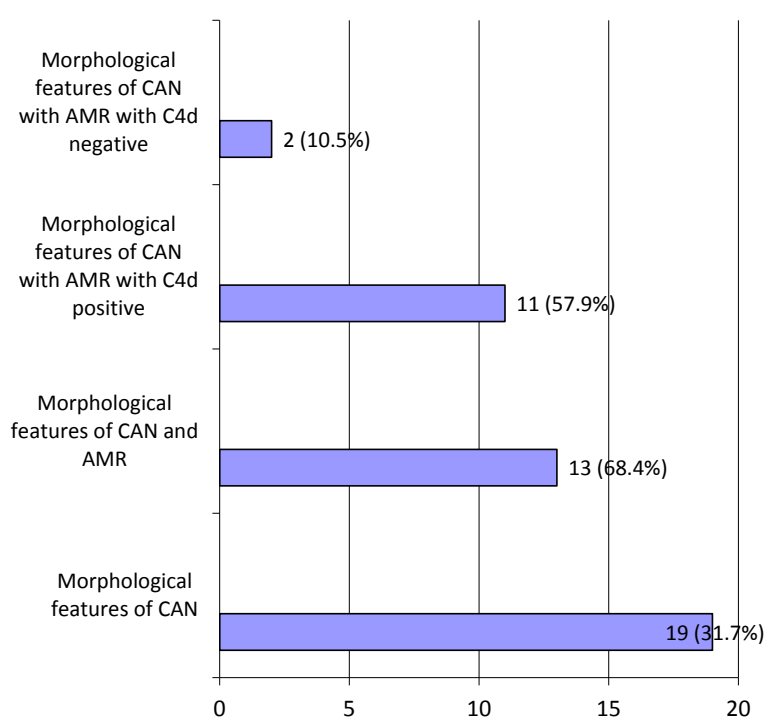

Figure 3. Distribution of acute antibody mediated rejection in late period of post transplantation in background of chronic allograft nephropathy.

in $3(5 \%)$ cases. These cases were also C4d positive. IgA nephropathy, acute pyelonephritis and extensive oxalate crystal deposition each in one $(1.7 \%)$ case.

\section{Discussion}

Long-term graft survival in renal transplantation strongly depends on events occurring early, particularly during first year after transplantation. A major event is an episode of acute rejection which has a major impact on long term performance of graft function. AMR should be considered in severe graft dysfunction setting, even when histopathologic features are equivocal (20).

Age range in our study was from 15 to 65 days with mean $34.6 \pm 11.8$. Patients in study conducted by Guduru et al had age range from 18 to 67 years (mean age 42.5 years) (11). The study by Ranjan et al had age ranges from 10 to 65 years with a mean of 34 years (6). Our cases had approximately similar age range.

In our study, 60 biopsies were evaluated with posttransplant period from 3 days to 5400 days (mean 33.8土 46.8). Nickeleit et al analyzed 398 indication allograft post-transplant biopsies obtained during seven days to 7165 days (21). Results of our study had similar time posttransplant duration.

Biopsies included in our study were over period of 2 years. Ranjan et al, Carpio et al, Botermans et al, Nickeleit et al, 

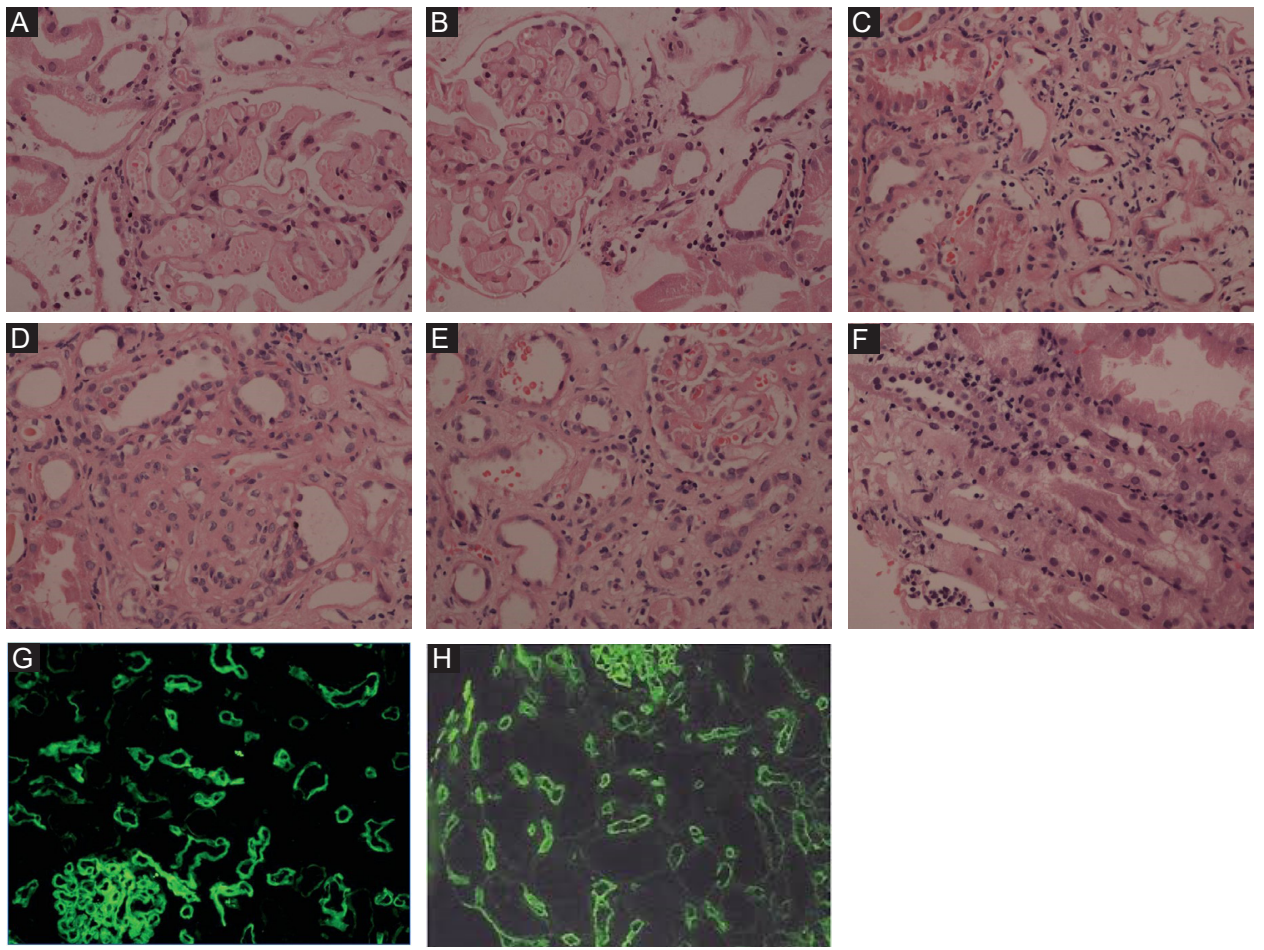

Figure 4. (A) Morphological features of acute antibody mediated rejection. Renal biopsy showing glomerulitis and mild degree of peritubular capillaritis (PC1). (B) Morphological features of acute antibody mediated rejection. Renal biopsy showing focal glomerulitis and moderate degree of peritubular capillaritis (PC2). (C) Morphological features of acute antibody mediated rejection. Renal biopsy showing marked degree of peritubular capillaritis (PC3). Tubular damage is also seen. (D) Morphological features of acute antibody mediated rejection, superimposed on chronic allograft nephropathy. Renal biopsy showing mild degree of peritubular capillaritis (PC1). (E) Morphological features of acute antibody mediated rejection, superimposed on chronic allograft nephropathy. Renal biopsy showing moderate degree of peritubular capillaritis (PC2) and focal glomerulitis. (F) Morphological features of acute antibody mediated rejection, superimposed on chronic allograft nephropathy. Renal biopsy showing marked degree of peritubular capillaritis (PC3). (G) C4d immunostaining. Glomerulus serves as a good internal control. Peritubular capillaries show diffuse circumferential deposits. (H) C4d immunostaining. Glomerulus serves as a good internal control. Peritubular capillaries show diffuse circumferential deposits.

and Larpparisuth et al performed studies over one year, 15 years, 9 years, 8 years and 8 years, respectively $(6,20-23)$. In this study, we evaluated 60 indication biopsies and demonstrated that a substantial fraction $(26.7 \%)$ of all biopsy-proven acute allograft rejection had a component of AMR as judged by morphology and C4d deposition in PTC. However, we also found that $6.25 \%$ of AMR cases would not have been recognized without $\mathrm{C} 4 \mathrm{~d}$ stain. These cases showed acute tubular injury. C4d staining of renal allograft biopsies is therefore essential in the recognition of AMR especially when features of only tubular injury are present. Similar findings are reported by Mauyyindi et al, who had analyzed 232 renal allograft biopsies and demonstrated $25 \%$ of C4d AMR cases without morphological features (24).

Our study showed $26.7 \%$ cases of C4d positive AMR, which fall in the spectrum of previous studies results. In Western countries, C4d varies from $17 \%$ to $60 \%$ among the indication biopsies. Regarding Asian population, only few prevalence studies had been conducted so far. In a Thai study, the prevalence of C4d was $16.4 \%$. Lower prevalence of $\mathrm{C} 4 \mathrm{~d}$ has been reported in indication biopsies in studies by Mengel et al (16) in 20.7\% and Cheunsuchon et al in $16.4 \%$. Out of these biopsies, $10.4 \%$ showed diffuse positivity and $5.9 \%$ had focal positivity (25). The reason of this variation includes type of biopsy, immunological risks for AMR, percentages of PTC positivity, time since transplant, case selection and techniques applied for immunostaining. In Harvard study, about $30 \%$ of all acute renal allograft rejections had a humoral component (7). In the study conducted in Brazil by Carpio et al, they showed $31 \%$ prevalence of C4d (22). C4d deposition had varied from $30 \%$ to $45 \%$ in other studies $(6,10,21,26,27)$. In the largest study to date, Nickeleit et al investigated a total of 398 diagnostic biopsies performed and found C4d positivity in 12 specimens (30\%) (21). Although our result is similar to this study, there is a significant difference in sample size.

We had 18 cases of acute rejection. Among them, 16 (26.7\%) cases had morphological features of AMR while 2 (11\%) cases of ACR. Out of 16 cases, 10 (62.5\%) showed C4d positivity, with further subdivision as $8(80 \%)$ diffuse and 2 (20\%) focal C4d positivity. Rests of cases were negative for C4d immunostaining. Botermans et al evaluated 151 patients which had a clinical suspicion of acute rejection. Among them, 128 (84.8\%) patients 
showed histological evidence of AMR, and only $12.5 \%$ $(n=16)$ showed C4d diffuse positivity by IF (20). In another study, conducted by Mauiyyedi et al, they had a sample size of 232 with 67 patients showing AMR (24). Out of them $75 \%$ had morphological evidence of AMR. Ludovico-Martins et al analyzed 20 cases with features of AMR. C4d deposition was found in $45 \%$ of their cases (28). In our study, stronger C4d positivity was associated with diffuse morphological features including peritubular capillaritis and glomerulitis. We found that focal versus diffuse C4d pattern was related to presence or absence of morphological features of AMR, respectively. Ranjan et al evaluated 132 core biopsies and C4d was performed in 126 of cases. AMR was diagnosed in $81 \%$ of total $45 \%$ of cases of rejection. Diffuse C4d was positive in $36.7 \%$ of total cases (6). Our results were similar to results of Ludovico-Martins et al and Mauiyyedi et al, but showed variation from Boterman et al and Ranjan et al's results. This difference may be due to a significant difference in sample size.

AMR is commonly accompanied by ACR i.e. these cases are classified as mixed ACR and AMR but AMR can occur in isolation. In literature, approximately $20 \%$ to $40 \%$ of ACR cases also have concurrent AMR. In our two cases, morphological features of both ACR and AMR were present. These cases were also C4d positive. Matignon et al conducted a study on 87 patients and found AMR in 29 and AMR with ACR in 18 (29). A study in Massachusetts general hospital and Harvard medical school found, ACR with AMR in $45 \%$ of cases (26). Concurrent presence of both AMR and ACR is associated with more severe graft dysfunction and treatment of both are different (30). Hence, it is important to look for both in a renal biopsy and report them separately.

According to Banff classification, extent of peritubular capillaritis should be mentioned in the report along with $\mathrm{C} 4 \mathrm{~d}$ result because the rate of $\mathrm{C} 4 \mathrm{~d}$ positive graft dysfunction was significantly higher if present along with peritubular capillaritis. Peritubular capillaritis is an important indicator of AMR in renal biopsies (31). In our study, 15 (25\%) cases had diffuse peritubular capillaritis and $8(13.3 \%)$ cases showed focal peritubular capillaritis. Glomerulitis was also present focally in $6(10 \%)$ cases and in diffuse pattern in 9 (15\%). Total of 16 cases had a good morphological evidence of AMR but only 10 showed diffuse C4d positivity. Kozakowski et al, studied 1322 renal biopsies with 329 showing peritubular capillaritis. Focal peritubular capillaritis was found in $10.5 \%$ of cases and diffuse peritubular capillaritis was in $14.4 \%$ of cases (32). Different studies reported the prevalence of peritubular capillaritis between $17 \%-46 \%(33,34)$. Results of diffuse peritubular capillaritis are quite close to comparison study but those of focal peritubular capillaritis were almost double of our results. All these studies had variation in total number of cases.

Out of 16 AMR cases, only 10 (62.5\%) showed C4d positivity. Eight cases showed diffuse PTC positivity for C4d and 2 (12.5\%) were focal C4d positive. Diffuse C4d deposition in renal allografts biopsies is associated with graft failure. A study done by Kedainis et al from Washington University, reviewed 368 indication biopsies from 301 transplant patients (35). In study by Kayler et al, 106 biopsies were included. C4d was positive in 40 and negative in 66 (36). These studies proposed that not only diffuse but also focal C4d positivity is a poor prognostic factor for AMR and graft survival. The impact of focal C4d positive biopsies should be considered in further treatment strategies.

We had one case in which C4d was positive with focal evidence of tubular damage but peritubular capillaritis or glomerulitis were absent. In their study, two patients had widespread C4d immunostaining in PTC. The study performed by Taheri et al, $17 \%$ of total 44 renal specimens, showed diffuse positivity for $\mathrm{C} 4 \mathrm{~d}$, with no microscopic features of AMR (24). They concluded that C4d immunostaining should be performed in every renal graft biopsy as features of AMR can be focal or even masked by other microscopic features. These cases can be missed if C4d staining was not performed. In Pakistan, C4d immunostaining was not available at every institution performing and evaluating renal allograft biopsies.

In our study we demonstrated $6.25 \%$ of AMR cases had diffuse C4d positivity with no morphological features of AMR. Similar findings were reported by various other studies, including a study Taheri et al; They reported $17 \%$ of 44 renal specimens with diffuse C4d positivity with no histological features of AMR (36). They concluded that C4d staining should be performed in all renal allograft biopsies and possibility of AMR should be considered even if its features are masked by other microscopic changes. In a study of Boston, it was mentioned that $25 \%$ of cases would have been missed if C4d immunostaining had not be performed (37). Therefore, it had been recommended in Banff criteria of AMR, to combine morphological features with C4d immunostaining in every renal biopsy. Even focal morphological features must be given weight age and mentioned in the final report.

Initially CAN was used which was a nonspecific term, as it can be the result of various conditions. In Banff 2005 schema it was replaced by interstitial fibrosis and tubular atrophy (IF/TA). If features of AMR are present on background of CAN, then these cases are categorized as chronic active antibody mediated rejection (38). It is reported that $\mathrm{C} 4 \mathrm{~d}$ serves as a reliable marker for detection of AMR in such cases (39). In our study, 29 cases were diagnosed as CAN. Out of these 10 had only features of CAN, two had features of CAN and morphological features of AMR, 11 had CAN, with morphological features of AMR and diffuse C4d positivity, one case of CAN, with morphological features of AMR and focal C4d positive. In studies performed during the early 21 st century, a frequency of AMR in background of CAN was 
reported in the range of $4.4 \%$ to $61 \%(21,24,40)$. In 2005 and onwards, AMR in background of CAN was observed in $5.1 \%$ to $50 \%$ of cases $(6,13,41-43)$. Yoon et al reported $5.1 \%$ of biopsies, and this difference is probably due to the inclusion of only protocol (42). Issa et al found C4d deposition in 24\% of biopsies (43). Previous studies by the same group had reported $\mathrm{C} 4 \mathrm{~d}$ immunodeposition in $25 \%$ and $36 \%$ of cases (44). In upcoming literature, complement independent i.e. C4d negative type of CAN has been supported (45). It had been emphasized to perform graft biopsy even in late post-transplant period to reach diagnosis. Currently in addition to therapy of AMR, antifibrinogenic agents had been developed and there is a hope that these can reverse fibrosis $(46,47)$.

In renal allografts, findings other than rejection can be seen and can also be a cause of graft dysfunction. In our 60 cases, ACR was documented in 7 (11.6\%) cases, cyclosporine drug toxicity in $5(8.3 \%)$ cases, tubulointerstitial nephritis in 5 $(8.3 \%)$ cases, tubular damage and necrosis $5(8.3 \%)$ cases. BK nephropathy was documented in 3 (5\%) cases. Each of IgA nephropathy, acute pyelonephritis and extensive oxalate crystal deposition were present in one (1.7\%) case. Taheri et al had acute tubular necrosis (ATN) in 27\%, CNI toxicity in $12.1 \%$ and $14.6 \%$ of cases had other findings including recurrence of primary glomerulonephritis, BK virus nephropathy and TIN and cortical infarction (37). Kulkarni et al had seven cases of ATN, CNI toxicity in 5 and BK virus nephropathy (13). As AMR can be the cause of graft dysfunction even many years of renal transplant, thus in every case, a biopsy must be submitted for C4d staining. This is important as morphological features can be masked due to any other concurrent etiology.

Donor specific antibodies level is a must in current Banff criteria of AMR in addition to morphological evidence and C4d positivity. In many previous cases DSA levels were available and results were compiled along with morphological features and C4d immunostaining pattern. We had C4d staining in $36.7 \%$ of total cases. In different studies $30 \%, 31 \%, 46 \%, 50 \%$ and $39.3 \%$ cases had C4d positivity $(10,22,48-50)$. DSA levels were not available in any of our cases. Our results were close to results of all these studies results, however, parameters which differed were sample size, lack of DSA levels, shorter duration posttransplantation and difference in C4d immunostaining techniques.

We analyzed C4d staining only by IF technique which is superior to IHC technique. Out of our 60 cases, C4d positivity was in $36.7 \%$ of cases including diffuse positivity in 43 and focal positivity in 2 cases. Carpio et al evaluated 146 renal biopsies. C4d staining was performed by IHC. Among them 31\% had C4d staining, with further sub-distribution as diffuse positivity in $14.7 \%$ and focal positivity in $16 \%$ (22). Worthington et al studied 92 cases and C4d was performed by immunohistochemistry. Among them 15\% and 24\% cases showed diffuse and focal C4d immunopositivity (51). Although total C4d positivity results are close, further subdivision into diffuse positivity and focal positivity show variation. Even though IHC had documented to provide appropriate results in renal transplant setting for C4d, IF is still considered as a gold standard (52). Hence, cases with focal C4d positivity by IHC should be evaluated by IF technique for definitive characterization.

\section{Conclusion}

C4d positivity favors AMR, however, it can be seen in absence of morphological features and results should be interpreted by taking in account other findings like donor specific antibody and endothelial activation markers. With the increasing use of $\mathrm{C} 4 \mathrm{~d}$ by transplant pathologists worldwide, several shortcomings of C4d have been identified and C4d appears to be a less sensitive marker than initially thought. Furthermore, molecular studies have provided insight suggestive of a complementindependent form of AMR.

\section{Limitations of the study}

Our study had few deficiencies including low sample count and lack of DSA levels. Hence, variability in results of our study while comparing it to other studies was seen. Previous studies had repeatedly proven that C4d staining is strongly related to DSA levels. However, emerging data show that AMR can be C4d negative and still relates to DSA levels. So additional work is required in this particular topic to see the actual trend of AMR in our region.

\section{Authors' contribution}

HN conceived the study and interpreted the light microscopy and immunofluorescent findings. HT collected, analyzed the data and prepared the manuscript. Both authors read, revised and approved the final manuscript.

\section{Conflicts of interest}

The authors declared no competing interests.

\section{Ethical considerations}

Ethical issues (including plagiarism, misconduct, data fabrication, falsification, double publication or submission, redundancy) have been completely observed by the authors.

\section{Funding/Support}

None.

\section{References}

1. Jha V. Current status of end-stage renal disease care in South Asia. Ethn Dis. 2009;19:S1-27-32.

2. Sakhuja V, Sud K. End-stage renal disease in India and Pakistan: burden of disease and management issues. Kidney Int Suppl. 2003:S115-8. doi: 10.1046/j.1523-1755.63. s83.24.x 2

3. Zuber K, Howard T, Davis J. Transplant in the 21st 
century. JAAPA. 2014;27:26-34. doi: 10.1097/01. JAA.0000455644.58683.e8.

4. Khan UF, Alam S, Bhatti N, Barki NU, Usman F, Bhatti TA, et al. Application of Banff criteria on cases of acute renal transplant rejection. J Rawal Med Coll. 2000;4:24-6.

5. Khalil Ullah, Iftikhar R, Moin S, Badsha S. Post-transplant complications. Pak J Med Res. 2003;42:174-8.

6. Ranjan P, Nada R, Jha V, Sakhuja V, Joshi K. The role of C4d immunostaining in the evaluation of the causes of renal allograft dysfunction. Nephrol Dial Transplant. 2008; 23:1735-41. doi: 10.1093/ndt/gfm843

7. Feucht HE. Complement C4d in graft capillaries -- the missing link in the recognition of humoral alloreactivity. Am J Transplant. 2003;3:646-52.

8. Pallardó Mateu LM, Sancho Calabuig A, Capdevila Plaza L, Franco Esteve A. Acute rejection and late renal transplant failure: risk factors and prognosis. Nephrol Dial Transplant. 2004;19 Suppl 3:iii38-42. doi: 10.1093/ndt/gfh1013 8.

9. Fior F, Nacchia F, Minicozzi A, Ghimenton C, Boschiero L. Focal C4d staining in peritubular capillaries and kidney graft survival: results of a retrospective Study. Transplant Proc. 2010;42:1095-7. doi: 10.1016/j.transproceed.2010.03.105 9

10. Nickeleit V, Zeiler M, Gudat F, Thiel G, Mihatsch MJ. Detection of the complement degradation product C4d in renal allografts: diagnostic and therapeutic implications. J Am Soc Nephrol. 2002;13:242-51.

11. Guduru SL, Kuruvilla S, Abraham G, Mathew M, Saravanan S. Diagnostic efficacy of C4d immunostaining in the detection of the humoral component of renal allograft rejection and therapeutic implications. Indian J Pathol Microbiol. 2009;52:345-8. doi: 10.4103/0377-4929.54990.

12. Kozakowski N, Regele H. Biopsy diagnostics in renal allograft rejection: from histomorphology to biological function. Transpl Int. 2009;22:945-53. doi: 10.1111/j.14322277.2009.00885.x.

13. Kulkarni P, Uppin MS, Prayaga AK, Das U, Murthy KVD. Renal allograft pathology with C4d immunostaining in patients with graft dysfunction. Indian J Nephrol. 2011; 21:239-44. doi: 10.4103/0971-4065.85481.

14. Solez K, Colvin RB, Racusen LC, Haas M, Sis B, Mengel $\mathrm{M}$, et al. Banff 07 classification of renal allograft pathology: updates and future directions. Am Transplant. 2008; 8:75360. doi: 10.1111/j.1600-6143.2008.02159.x.

15. Correa RRM, Machado JR, da Silva MV,Helmo FR, Guimaraes CSO, Rocha LP, et al. The importance of C4d in biopsies of kidney transplant recipients. Clin Dev Immunol. 2013;2013:678180. doi: 10.1155/2013/678180.

16. Mengel M, Sis B, Haas M, Colvin RB, Halloran PF, Racusen LC, et al.; Banff meeting report writing committee. Banff 2011 Meeting report: new concepts in antibodymediated rejection. Am J Transplant. 2012;12:563-70. doi: 10.1111/j.1600-6143.2011.03926.x.

17. Sis B, Mengel M, Haas M, Colvin RB, Halloran PF, Racusen LC, Solez K, et al. Banff'09 meeting report: antibody mediated graft deterioration and implementation of Banff working groups. Am J Transplant. 2010;10:464-71. doi: 10.1111/j.1600-6143.2009.02987.x.

18. Cohen D, Colvin RB, Daha MR, Drachenberg CB, Haas $\mathrm{M}$, Nickeleit V, et al. Pros and cons for C4d as a biomarker. Kidney Int. 2012;81:628-39. doi: 10.1038/ki.2011.497.

19. Watschinger B, Pascual M. Capillary C4d deposition as a marker of humoral immunity in renal allograft rejection. JASN. 2002;13:2420-3.
20. Botermans JM, de Kort H, Eikmans M, Koop K, Baelde HJ, Mallat MJK, et al. C4d staining in renal allograft biopsies with early acute rejection and subsequent clinical outcome. Clin J Am Soc Nephrol. 2011;6:1207-13. doi: 10.2215/ CJN.07820910.

21. Nickeleit V1, Mihatsch MJ. Kidney transplants, antibodies and rejection: is C4d a magic marker? Nephrol Dial Transplant. 2003;18:2232-9.

22. Carpio VN, Rech C, Eickhoff EI, Pegas KL, Edelweiss MI, Gonçalves LF, et al. Clinical and pathological correlations of C4d immunostaining and its influence on the outcome of kidney transplant recipients. J Bras Nefrol. 2011; 33:329-37.

23. Larpparisuth $\mathrm{N}$, Vongwiwatana A, Vareesangthip $\mathrm{K}$, Cheunsuchon B, Parichatikanon P, Premasathian N. Clinicopathologic features and treatment response of early acute antibody-mediated rejection in Thai kidney transplant recipients: a single-center experience. Transplant Proc. 2014;46:474-6. doi: 10.1016/j.transproceed.2013.12.022.

24. Mauiyyedi S, Crespo M, Collins AB, Schneeberger EE, Pascual MA, Saidman SL, et al. Acute humoral rejection in kidney transplantation: II. Morphology, immunopathology, and pathologic Classification. J Am Soc Nephrol. 2002;13:779-87.

25. Cheunsuchon B, Vongwiwatana A, Premasathian N, Shayakul C, Parichatikanond P. The prevalence of C4dpositive renal allografts in 134 consecutive biopsies in Thai patients. Tansplant Proc. 2009; 41:3697-700. doi: 10.1016/j. transproceed.2009.04.015

26. Herzenberg AM, Gill JS, Djurdjev O, Magil AB. C4d deposition in acute rejection: an independent long-term prognostic factor. J Am Soc Nephrol 2002;13:234-41.

27. Regele $\mathrm{H}$, Exner M, Watschinger B, Wenter C, Wahrmann $\mathrm{M}$, Osterreicher $\mathrm{C}$, et al. Endothelial C4d deposition is associated with inferior kidney allograft outcome independently of cellular rejection. Nephrol Dial Transplant. 2001;16:2058-66.

28. Ludovico-Martins H, Silva C, Teodoro WR, Martini Filho D, Noronha IL. Analysis of different staining techniques for c4d detection in renal allograft biopsies. Transplant Proc. 2009;41:862-5. doi: 10.1016/j.transproceed.2009.01.073.

29. Matignon M, Muthukumar T, Seshan SV, Suthanthiran M, Hartono C. Concurrent Acute cellular rejection is an independent risk factor for renal allograft failure in patients with C4d positive antibody-mediated rejection. Transplantation. 2012;94:603-611. doi: 10.1097/ TP.0b013e31825def05

30. Jennette JC, Oslon JL, Silva FG, D'Agati VD, eds. Heptinstall's Pathology of the Kidney. Philadelphia: Wolters Kluwer; 2015.

31. Jin J, Xu Y, Wang H, Huang H, He Q, Wu P, et al. Peritubular capillaritis in early renal allograft dysfunction is an indicator of acute rejection. Transplant Proc. 2013;45:163-71. doi: 10.1016/j.transproceed.2012.08.018.

32. Kozakowski N, Herkner H, Bohmig GA, Regele H, Kornauth C, Bond G, Kikic Z. The diffuse extent of peritubular capillaritis in renal allograft rejection is an independent risk factor for graft loss. Kidney Int. 2015;88:332-40. doi: 10.1038/ki.2015.64.

33. Aita K, Yamaguchi Y, Horita S. Peritubular capillaritis in early renal allograftis associated with the development of chronic rejection and chronic allograft nephropathy. Clin Transplant. 2005;19:20-6. doi: 10.1111/j.13990012.2005.00400.x. 
34. Gibson IW, Gwinner W, Bröcker V, Sis B, Riopel J, Roberts IS, et al. Peritubular capillaritis in renal allografts: prevalence, scoring system, reproducibility and clinicopathological correlates. Am J Transplant 2008;8:81925. doi: 10.1111/j.1600-6143.2007.02137.x.

35. Kedainis RL, Koch MJ, Brennan DC, Liapis H. Focal $\mathrm{C} 4 \mathrm{~d}+$ in renal allografts is associated with the presence of donor-specific antibodies and decreased allograft survival. Am J Transplant. 2009;9:812-9. doi: 10.1111/j.16006143.2009.02555.x.

36. Kayler LK, Kiss L, Sharma V, Mohanka R, Zeevi A, Girnita A, et al. Acute renal allograft rejection: diagnostic significance of focal peritubular capillary C4d. Transplantation. 2008; 85:813-20. doi: 10.1097/TP.0b013e3181669194.

37. Taheri D, Talebi A, Taghaodi M, Fesharakizadeh M, Mortazavi M, Azhir A, et al. Pathological diagnosis of antibody-mediated rejection in renal allograft without c4d staining, how much reliable? Adv Biomed Res. 2012;1:40. doi: $10.4103 / 2277-9175.100139$.

38. Haas M, Sis B, Rascusen LC, Solez K, Glotz D, Colvin RB, et al. Banff 2013 Meeting Report: Inclusion of C4d-negative antibody-mediated rejection and antibody-associated arterial lesions. Am J Transplant. 2014;14:273-83. doi: 10.1111/ajt.12590.

39. Böhmig GA, Exner M, Habicht A, Schillinger M, Lang U, Kletzmayr J, et al. Capillary C4d deposition in kidney allografts: a specific marker of alloantibody-dependent graft injury. J Am Soc Nephrol. 2002;13:1091-9.

40. Mengel M, Bogers J, Bosmans JL, Serón D, Moreso F, Carrera $\mathrm{M}$, et al. Incidence of $\mathrm{C} 4 \mathrm{~d}$ stain in protocol biopsies from renal allografts: results from a multicenter trial. Am J Transplant. 2005;5:1050-6. doi: 10.1111/j.16006143.2005.00788.x.

41. Akalin E, Dinavahi R, Dikman S, de Boccardo G, Friedlander R, Schroppel B, et al. Transplant glomerulopathy may occur in the absence of donor-specific antibody and C4d staining. Clin J Am Soc Nephrol. 2007;2:1261-7. doi: 10.2215/ CJN.02420607.

42. Yoon HE, Kim SH, Kim JY. Clinical significance of C4d deposits in stable renal allografts in the early post transplant period. Clin Transplant. 2009;23:812-8.
43. Issa N, Cosio FG, Gloor JM, Sethi S, Dean PG, Moore SB, et al. Transplant glomerulopathy: risk and prognosis related to anti-human leukocyte antigen class II antibody levels. Transplantation. 2008;86:681-5. doi: 10.1097/ TP.0b013e3181837626.

44. Racusen LC, Colvin RB, Solez K, Mihatsch MJ, Halloran PF, Campbell PM, et al. Antibody-mediated rejection criteria an addition to the Banff 97 classification of renal allograft rejection. Am J Transplant. 2003;3:708-14.

45. Sis B, Jhangri GS, Bunnag S, Allanach K, Kaplan B, Halloran $\mathrm{PF}$. Endothelial gene expression in kidney transplants with alloantibody indicates antibody-mediated damage despite lack of C4d staining. Am J Transplant. 2009;9:2312-23. doi: 10.1111/j.1600-6143.2009.02761.x.

46. Hewitson TD. Renal tubulointerstitial fibrosis: common but never simple. Am J Physiol Renal Physiol 2009;296:F123944.

47. Racusen LC. Protocol transplant biopsies in kidney allografts: Why and when are they indicated? Clin J Am Soc Nephrol. 2006;1:144-7.

48. Kara M, Demir F, Ata P, Ozel L, Gumrukcu G, Unal $\mathrm{E}$, et al. The impact of C4d staining as a humoral injury marker. Transplant Proc. 2012;44:1694-6. doi : 10.1016/j. transproceed.2012.05.029.

49. Lederer SR, Kluth-Pepper B, Schneeberger H, Albert E, Land W, Feucht HE. Impact of humoral alloreactivity early after transplantation on the long-term survival of renal allografts. Kidney Int. 2001;59:334-41.

50. Shimizu T1, Tanabe T, Omoto K, Ishida H, Tanabe K. Clinicopathologic analysis of acute vascular rejection cases after renal transplantation. Transplant Proc. 2012;44:230-5. doi: 10.1016/j.transproceed.2011.11.002.

51. Troxell ML, Weintraub LA, Higgins JP, Kambham N. Comparison of C4d immunostaining methods in renal allograft biopsies. Clin J Am Soc Nephrol. 2006;1:583-91.

52. Worthington JE, McEwen A, McWilliam LJ, Picton ML, Martin S. Association between C4d staining in renal transplant biopsies, production of donor-specific HLA antibodies, and graft outcome. Transplantation. 2007;83:398-403.

Copyright ( $\odot 2018$ The Author(s); Published by Nickan Research Institute. This is an open-access article distributed under the terms of the Creative Commons Attribution License (http://creativecommons.org/licenses/by/4.0), which permits unrestricted use, distribution, and reproduction in any medium, provided the original work is properly cited. 